\title{
GENERALIZATION OF MENGER'S RESULT ON THE STRUCTURE OF LOGICAL FORMULAS
}

\author{
DAL CHARLES GERNETH ${ }^{1}$
}

Menger's paper ${ }^{2}$ gives necessary and sufficient conditions that an expression containing sentential variables and unary and binary sentential connectives be a formula in the Eukasiewicz notation. This paper extends his result to expressions containing $n$-ary symbols for all $n \geqq 0$. Sentential variables and constants are treated as the case $n=0$.

An expression is a sequence $s_{1} \cdots s_{k}$ such that $s_{i}$ for $i=1, \cdots, k$ is an $n$-ary symbol for some $n$. An initial segment of such an expression is an expression $s_{1} \cdots s_{i}$ where $i<k$; a terminal segment is an expression $s_{t} \cdots s_{k}$ where $t>1$. A formula is a sequence $s z_{1} \cdots z_{n}$ where $s$ is an $n$-ary symbol, and $z_{1}, \cdots, z_{n}$ are formulas. The measure $[s]$ of an $n$-ary symbol $s$ is $n-1$. The measure $\left[s_{1} \cdots s_{k}\right]$ of an expression $s_{1} \cdots s_{k}$ is $\left[s_{1}\right]+\cdots+\left[s_{k}\right]$.

TheOREM. Necessary and sufficient conditions that an expression $x=s_{1} \cdots s_{k}$ be a formula are:

$$
[y] \geqq 0 \text { for each initial segment } y \text { of } x,
$$

and

$$
[x]=-1 .
$$

Proof. Suppose $s$ is an $n$-ary symbol, $z_{1}, \cdots, z_{h}, h \geqq 0$ are formulas, $z$ is an initial segment of a formula $z_{h+1}$, and $z_{1}, \cdots, z_{h+1}$ satisfy (1) and (2). Then

$$
\begin{aligned}
{\left[s z_{1} \cdots z_{h}\right] } & =[s]+\left[z_{1}\right]+\cdots+\left[z_{h}\right] \\
& =(n-1)-1-\cdots-1=n-1-h
\end{aligned}
$$

and

$$
\left[s z_{1} \cdots z_{h} z\right]=n-1-h+[z] \geqq n-1-h .
$$

PRoOF OF NeCEssity. Let $x=s z_{1} \cdots z_{n}$ be a formula where by the induction hypothesis $s$ is an $n$-ary symbol and $z_{1}, \cdots, z_{n}$ are formulas

Received by the editors August 14, 1947.

1 Paper suggested by Dr. J. C. C. McKinsey and Miss Helen Dayton of Oklahoma Agricultural and Mechanical College.

2 Menger, Karl, Eine elementare Bemerkung uber die Struktur logischer Formeln, Ergebnisse eines mathematischen Kolloquiums, vol. 3, 1930-1931, pp. 22-23. 
satisfying (1) and (2). An initial segment $y$ of $x$ has one of the forms (3) or (4), with $h<n$ so that $[y] \geqq 0$, and (3) applies to $x$ with $h=n$, giving $[x]=-1$.

Proof of sufficiency. An expression of length one is a formula by (2) and the definition. Suppose all expressions of length less than $k$ satisfying (1) and (2) are formulas, and let $x=s_{1} \cdots s_{k}$ be an expression of length $k>1$ satisfying (1) and (2).

Lemma. Starting at any symbol $s_{i}$ of $x, 1 \leqq i \leqq k$, there is a unique segment satisfying (1) and (2).

Proof. For any terminal segment $s_{t} \cdots s_{k}$ of $x$, we have $\left[s_{1} \cdots s_{t-1}\right]+\left[s_{t} \cdots s_{k}\right]=[x]$ or $\left[s_{t} \cdots s_{k}\right] \leqq-1$ by (1) and (2). Thus, for any symbol $s_{i}, 1 \leqq i \leqq k$, there is a symbol $s_{j}, i \leqq j \leqq k$, such that $\left[s_{i} \cdots s_{j}\right]=-1$. For each integer $i$, only the smallest such integer $j$ provides a segment $s_{i} \cdots s_{j}$ satisfying (1) as well as (2).

By (1), $s_{1}$ is a connective $s$ for some $n>0$. The lemma may be applied, starting at $s_{2}$, to exhaust the symbols of $x$ by constructing consecutive segments $z_{1}, \cdots, z_{h}$, each a formula by the induction hypothesis. Now for $x=s z_{1} \cdots z_{h}$, by (2) and (3), $[x]=-1=n-1-h$ or $h=n$; hence $x$ is a formula.

This completes the proof of the theorem.

CoROLlARY. In any formula, starting at a given symbol, there is a unique consecutive part which is a formula.

Sherman, Tex. 